\title{
ACYCLIC MODELS AND DE RHAM'S THEOREM
}

\author{
BY \\ AMASA FORRESTER
}

\section{INTRODUCTION}

The concept of acyclic models, developed by Eilenberg and MacLane [2], appears to be one of the most convenient tools of algebraic topology. It is the purpose of this paper to describe a generalization of the theory of Eilenberg and MacLane, and to apply the theory to proving de Rham's theorem. The generalized theory has other applications; in particular the proof of the spectral sequence theorem due to Gugenheim and Moore [4] could be slightly simplified by the results of this paper.

The reader is assumed to know the theory of functors and categories; the general reference for this topic will be Eilenberg and Steenrod [3, Chapter IV, pp. 108-113].

The first two chapters are devoted to establishing the existence or uniqueness of chain and cochain maps; the third chapter is devoted to cup products. In the fourth chapter the singular $C^{r}$ cubical cohomology theory is defined, and it is shown that for paracompact manifolds the result is independent of $r$. In the last chapter the de Rham cohomology is defined and shown to be isomorphic to the singular cohomology (de Rham's theorem) by means of the Stokes' map $\int_{*}$ (integration over chains); it is also shown that the Stokes' map preserves products, i.e., that $\int_{*}(\alpha \beta)=\left(\int_{*} \alpha\right) \cup\left(\int_{*} \beta\right)$. The relation of acyclic models to products will be treated at length in another paper.

\section{Chapter I. Model theory}

In this chapter the abstract concept of a model theory and of a representable functor is defined. A general method of obtaining model theories, due to Gugenheim and Moore [4], is described. Two points are of particular interest: the concept of model theory is given in a natural, or functorial, form; and the concept of semi-representability is introduced. Semi-representability is decisive in the proof of the de Rham theorem developed later.

1.1. Model theories. If $a$ and $B$ are categories, $F(a, B)$ will denote the category of covariant functors from $Q$ to $B$ : the objects of $F(Q, B)$ are the covariant functors $T: Q \rightarrow B$, and the maps $\Phi: T_{1} \rightarrow T_{2}$ of $F(Q, B)$ are the natural transformations $\Phi$ of covariant functors $T_{1}: Q \rightarrow ß, i=1,2$.

Rather than introduce an analogous concept for contravariant functors, we will utilize the concept of the dual of a category. If $a$ is a category, then the dual $a^{*}$ of $a$ is a category having the same objects and maps as $a$, but such that order is reversed in $Q^{*}$, i.e., if $A$ and $A^{\prime}$ are objects of $a$ and $a^{*}$

Received by the editors August 31, 1955. 
and $f$ is a map of these two categories, then $f: A \rightarrow A^{\prime}$ in $Q^{*}$ if and only if $f: A^{\prime} \rightarrow A$ in $a$; and if $f, g$ are maps of the two categories whose composition is defined, then the map of $Q$ denoted by $f g$ is denoted by $g f$ in $Q^{*}$.

If $Q$ and $B$ are categories, then $F\left(Q^{*}, Q\right)$ may be regarded as the category whose objects are contravariant functors from $Q$ to $B$ and whose maps are natural transformations of such contravariant functors.

An additive category is a category such that for every pair of objects $A, B$ of the category, the set of maps $f: A \rightarrow B$ in the category is assigned the structure of an abelian group, so that if $f, f_{1}, f_{2}: A \rightarrow B$ and $g, g_{1}, g_{2}: B \rightarrow C$ are maps of the category, then $g\left(f_{1}+f_{2}\right)=g f_{1}+g f_{2}$ and $\left(g_{1}+g_{2}\right) f=g_{1} f+g_{2} f$.

If $B$ is an additive category, then for any category $Q$ it is possible to make $F(Q, \beta)$ into an additive category in the following way: let $T_{1}, T_{2}: Q \rightarrow B$ be covariant functors and $\Phi, \Psi: T_{1} \rightarrow T_{2}$ be transformations; then $\Phi+\Psi$ is to be the natural transformation $T_{1} \rightarrow T_{2}$ such that for each object $A$ of $Q,(\Phi+\Psi)(A)$ $=\Phi(A)+\Psi(A)$. It is clear that $F(Q, B)$ is then an additive category.

Let now $Q, B$ be additive categories; an additive functor $R: Q \rightarrow B$ is a functor such that $R\left(f_{1}+f_{2}\right)=R\left(f_{1}\right)+R\left(f_{2}\right)$ for maps $f_{1}, f_{2}: A \rightarrow A^{\prime}$ in $Q$. It is clear that $R$ is a homomorphism of the group in $a$ of maps of $A$ into $A^{\prime}$, into the groups of maps in $B$ of maps of $R(A)$ into $R\left(A^{\prime}\right)$, or of $R\left(A^{\prime}\right)$ into $R(A)$ (depending on whether $R$ is covariant or contravariant). In particular, $R$ maps the zero of each such group into the zero of the image group.

In what follows we will use a particular additive category, the category of $\Lambda$-modules $G_{\Lambda}$; much of what will be done can be extended to more general additive categories. Throughout this chapter $\Lambda$ will denote a fixed associative ring with unit, and $g_{\Lambda}$ will denote the category of left $\Lambda$-modules and $\Lambda$-homomorphisms. If $\phi, \psi: G_{1} \rightarrow G_{2}$ are $\Lambda$-homomorphisms, let $\phi+\psi: G_{1} \rightarrow G_{2}$ be defined by $(\phi+\psi)(g)=\phi(g)+\psi(g)$ for $g \in G_{1}$; then it is clear that $G_{\Lambda}$ is an additive category, and thus that $F\left(Q, \Theta_{\Lambda}\right)$ is an additive category for any category $Q$.

Definition. A direct model theory on a category $Q$ is a pair $(R, \Gamma)$ composed of an additive covariant functor $R: F\left(Q, g_{\Lambda}\right) \rightarrow F\left(Q, G_{\Lambda}\right)$ and a natural transformation $\Gamma: R \rightarrow I$ of $R$ into the identity functor $I: F\left(Q, \mathcal{G}_{\Lambda}\right) \rightarrow F\left(Q, \mathcal{G}_{\Lambda}\right)$. An inverse model theory on $Q$ is a pair $(R, \Gamma)$ composed of an addlitive covariant functor $R: F\left(\alpha, \mathcal{S}_{\Lambda}\right) \rightarrow F\left(\alpha, \mathcal{S}_{\Lambda}\right)$ and a natural transformation $\Gamma: I \rightarrow R$ of the identity functor $I$ into the functor $R$.

1.2. Categories with models. Before dealing with the theory of models, it will be convenient to describe how model theories may be obtained; the scheme described below is due to Gugenheim and Moore [4].

A category with models is a system $(a, \mathfrak{T}, S)$ such that:

(i) $a$ is a category;

(ii) $\mathfrak{T}$ is a set of objects of $\alpha$, the elements of $\mathscr{N}$ being called models;

(iii) For each object $A$ of $a$ there is given a set $S(A)$ of maps $u: M \rightarrow A$ in a mapping models $M$ in $\mathfrak{T}$ into $A$ (we will write $(M, u) \in S(A)$ if $u: M \rightarrow A$ is in $S(A)$ ); 
(iv) If $u \in S(A)$ and $f: A \rightarrow B$ is a map of $a$, then $f u \in S(B)$.

REMARK. If each $S(A)$ consists of all maps $u: M \rightarrow A$ of models $M$ in $\mathfrak{T}$ into $A$, then (iii), and (iv) are also satisfied; we speak in this case of a category with models $(Q, \mathfrak{T})$. This case is that originally considered by Eilenberg and MacLane [2].

If $(Q, \mathfrak{T}, S)$ is a category with models, we can define a direct model theory $(R, \Gamma)$ on $Q$ and an inverse model theory $\left(R^{*}, \Gamma^{*}\right)$ on $Q^{*}$; these model theories will be called the model theories associated with $(Q, \mathfrak{T}, S)$. As a preliminary we make several notational definitions.

If $G$ is a $\Lambda$-module and $x$ is some object, $(G, x)$ will denote the group whose elements are pairs $(g, x), g \in G$, with $\left(g_{1}, x\right)+\left(g_{2}, x\right)=\left(g_{1}+g_{2}, x\right)$. If $T: Q \rightarrow \mathcal{G}_{\Lambda}$ is a functor and $f: A \rightarrow B$ is a map of $a, T(A, f)$ will denote the module $(T(A), f)$. If $G_{\alpha}$ is a $\Lambda$-module for $\alpha$ in a set $\Delta$, then $\sum_{\alpha \in \Delta} G_{\alpha}$ will denote the direct sum of the $G_{\alpha}$ and $\prod_{\alpha \in \Delta} G_{\alpha}$ will denote the direct product; an element $\gamma$ of $\prod_{\alpha \in \Delta} G_{\alpha}$ will be considered as a function on $\Delta$ such that $\gamma(\alpha) \in G_{\alpha}$ for $\alpha \in \Delta$.

Let $T: Q \rightarrow G_{\Lambda}$ be a covariant functor; we define a covariant functor $\hat{T}: Q \rightarrow \mathcal{G}_{\Lambda}$ and a transformation $\Gamma_{T}: \hat{T} \rightarrow T$ in the following way. If $A$ is an object of $a$, set $\hat{T}(A)=\sum_{(M, \mu) \in S(A)} T(M, u)$; if $f: A \rightarrow B$ is a map of $a, \hat{T}(f)$ is the homomorphism $\hat{T}(A) \rightarrow \hat{T}(B)$ defined by setting $\hat{T}(f)(m, u)=(m, f u)$ for $m \in T(M)$. Then it is easy to see that $\hat{T}$ is a covariant functor. Let $\Gamma_{T}(A)$ be the homomorphism $\hat{T}(A) \rightarrow T(A)$ defined by $\Gamma_{T}(A)(m, u)=T(u) m$ for $m \in M$; then $\Gamma_{T}$ is a transformation $\hat{T} \rightarrow T$. Finally, if $\Phi: T_{1} \rightarrow T_{2}$ is a transformation of covariant functors $T_{1}, T_{2}: Q \rightarrow \mathcal{G}_{\Lambda}$, let $\widehat{\Phi}(A)$ be the homomorphism defined by $\widehat{\Phi}(A)(m, u)=(\Phi(M) m, u)$ for $m \in T_{1}(M)$; then $\widehat{\Phi}$ is a transformation $\hat{T}_{1} \rightarrow \hat{T}_{2}$. Setting $R(T)=\hat{T}, R(\Phi)=\widehat{\Phi}$, and $\Gamma(T)=\Gamma_{T}$ then defines a covariant model theory $(R, \Gamma)$ on $Q$.

Let now $T: Q \rightarrow \mathrm{G}_{\Lambda}$ be a contravariant functor. We will define a functor $\hat{T}: Q \rightarrow \mathcal{G}_{\Lambda}$ and a transformation $\Gamma_{T}^{*}: T \rightarrow \hat{T}$. Let $\hat{T}(A)=\prod_{(M, \mu) \in S(A)} T(M, u)$; if $f: A \rightarrow B$ is a map, let $\hat{T}(f)$ be the homomorphism $\hat{T}(B) \rightarrow \hat{T}(A)$ defined by setting, for $\gamma$ an element of $\hat{T}(B)$ and $u$ in $S(A),(\hat{T}(f) \gamma)(u)=\gamma(f u)$. Then it is easy to see that $\hat{T}$ is a contravariant functor. Let $\Gamma_{T}^{*}(A)$ be the homomorphism $T(A) \rightarrow T(A)$ defined by $\left(\Gamma_{T}^{*}(A) a\right)(u)=T(u) a$ for $a \in T(A)$ and $u \in S(A)$; then $\Gamma_{T}^{*}$ is a transformation $T \rightarrow \hat{T}$. Finally, if $\Phi: T_{1} \rightarrow T_{2}$ is a transformation of contravariant functors $T_{1}, T_{2}: a \rightarrow g_{\Lambda}$, let $\widehat{\Phi}(A)$ be the homomorphism $\hat{T}_{1}(A) \rightarrow \hat{T}_{2}(A)$ defined by $(\widehat{\Phi}(A) \gamma)(u)=\Phi(M) \gamma(u)$ for $(M, u) \in S(A)$ and $\gamma$ an element of $\hat{T}_{1}(A)$; then $\Phi$ is a transformation $\hat{T}_{1} \rightarrow \hat{T}_{2}$. Setting $R^{*}(T)=\hat{T}, R^{*}(\Phi)=\widehat{\Phi}$, and $\Gamma^{*}(T)=\Gamma_{T}^{*}$ then defines an inverse model theory $\left(R^{*}, \Gamma^{*}\right)$ on $Q^{*}$.

1.3. Representations. The concept of model theories is of use mainly in connection with representable or semi-representable functors, which we will now define.

Definition. Let 


$$
(R, \Gamma) \text { be a }\left\{\begin{array}{l}
\text { direct } \\
\text { inverse }
\end{array}\right. \text { model theory }
$$

on a category $a$, and let $T: Q \rightarrow g_{\Lambda}$ be a covariant functor. Then $T$ is said to be representable if there is a transformation

$$
\left\{\begin{array} { l } 
{ \chi : T \rightarrow R ( T ) } \\
{ \chi : R ( T ) \rightarrow T }
\end{array} \quad \text { such that } \left\{\begin{array}{l}
\Gamma(T) \chi: T \rightarrow T \\
\chi \Gamma(T): T \rightarrow T
\end{array}\right.\right.
$$

is the identity transformation; is called a representation of $T$. The functor $T$ is called semi-representable if for each object $A$ of $a$ there is a $\Lambda$-homomorphism

$$
\left\{\begin{array} { r l } 
{ \overline { \chi } ( A ) : T ( A ) } & { \rightarrow R ( T ) ( A ) } \\
{ \overline { \chi } ( A ) : R ( T ) ( A ) } & { \rightarrow T ( A ) }
\end{array} \text { such that } \left\{\begin{array}{l}
\Gamma(T)(A) \bar{\chi}(A): T(A) \rightarrow T(A) \\
\bar{\chi}(A) \Gamma(T)(A): T(A) \rightarrow T(A)
\end{array}\right.\right.
$$

is the identity homomorphism for each object $A$ of $Q$; the function $\bar{\chi}$ is called a semi-representation of $T$. Clearly every representable functor is semirepresentable.

Proposition 1. Let $(R, \Gamma)$ be a direct or inverse model theory on a category $Q$, and let $T, T_{1}: Q \rightarrow \mathcal{G}_{\Lambda}$ be covariant functors. Let $\xi: T \rightarrow T_{1}, \eta: T_{1} \rightarrow T$ be transformations such that $\xi_{\eta}: T_{1} \rightarrow T_{1}$ is the identity transformation. Then if $T$ is representable (or semi-representable) so is $T_{1}$.

The reader is referred to Eilenberg and MacLane [2] for a proof which applies to the present proposition.

1.4. Products of model theories. Let $\left(R_{1}, \Gamma_{1}\right)$ and $\left(R_{2}, \Gamma_{2}\right)$ be model theories on a category $Q$, both direct or both inverse. The product $(R, \Gamma)$ of the two model theories is a model theory direct or inverse with $\left(R_{1}, \Gamma_{1}\right)$ and $\left(R_{2}, \Gamma_{2}\right)$, defined in the following way. If $T$ is a covariant functor, $R(T)(A)$ is to be the direct sum $R_{1}(T)(A)+R_{2}(T)(A)$ for each object $A$; if $\Phi: T_{1} \rightarrow T_{2}$ is a transformation of covariant functors, $R(\Phi)$ is to be the transformation which assigns to the object $A$ the homomorphism $R(\Phi)(A): R\left(T_{1}\right)(A)$ $\rightarrow R\left(T_{2}\right)(A)$ defined by $R(\Phi)(A)\left(a_{1}, a_{2}\right)=\left(R_{1}(\Phi) a_{1}, R_{2}(\Phi) a_{2}\right)$ for $a_{1} \in R_{1}\left(T_{1}\right)(A)$ and $a_{2} \in R_{2}\left(T_{1}\right)(A)$. If the theories are direct, $\Gamma$ assigns to $T$ and $A$ the homomorphism $\Gamma(T)(A): R(T)(A) \rightarrow T(A)$ defined by

$$
(\Gamma(T)(A))\left(a_{1}, a_{2}\right)=\Gamma_{1}(T)(A) a_{1}+\Gamma_{2}(T)(A) a_{2}
$$

for $a_{1} \in R_{1}(T)(A)$ and $a_{2} \in R_{2}(T)(A)$. If both theories are inverse, $\Gamma$ assigns to $T$ and $A$ the homomorphism $\Gamma(T)(A): T(A) \rightarrow R(T)(A)$ defined by $(\Gamma(T)(A)) a$ $=\left(\Gamma_{1}(T)(A) a, \Gamma_{2}(T)(A) a\right)$ for $a \in T(A)$.

Proposition 2. Let $\left(R_{1}, \Gamma_{1}\right)$ and $\left(R_{2}, \Gamma_{2}\right)$ be model theories on a category $a$, both direct or both inverse, and let $(R, \Gamma)$ be their product. If a functor $T$ is repre- 
sentable (or semi-representable) for either $\left(R_{1}, \Gamma_{1}\right)$ or $\left(R_{2}, \Gamma_{2}\right)$, then $T$ is representable (or semi-representable) for $(R, \Gamma)$.

The proof is trivial.

\section{Chapter II. Acyclic models}

In this chapter the concept of models is applied to homology and cohomology. After some preliminary definitions, the fundamental theorems of Eilenberg and MacLane [2] are established (Theorems 5, 6 and 7). In addition, two new theorems dealing with semi-representability are established. Theorem 9B is basically the proof of de Rham's theorem.

2.1. Chain and cochain functors. We will first define the categories $\partial g_{\Delta}$ and $\delta g_{\Delta}$ of chain and cochain complexes.

A chain complex is a $\Lambda$-module $G$ with a $\Lambda$-homomorphism $\partial: G \rightarrow G$ such that $\partial^{2}=0$, and a direct sum decomposition into submodules $G=\sum_{n} G_{n}(n$ running through the integers) such that $\partial G_{n} \subseteq G_{n-1}$; the map $G_{n} \rightarrow G_{n-1}$ induced by $\partial$ is denoted by $\partial_{n}$. A chain map $f: G \rightarrow G^{\prime}$ is a $\Lambda$-homomorphism such that $f \partial=\partial f$ and $f G_{n} \subseteq G_{n}^{\prime}$; the map $G_{n} \rightarrow G_{n}^{\prime}$ induced by $f$ is denoted by $f_{n}$. The category of chain complexes and chain maps will be denoted by $\partial \mathcal{G}_{\Lambda}$.

A cochain complex is a $\Lambda$-module $G$ with a $\Lambda$-homomorphism $\delta: G \rightarrow G$ such that $\delta^{2}=0$, and a direct product decomposition into submodules $G=\prod_{n} G_{n}$ such that $\delta G_{n} \subseteq G_{n+1}$; the map $G_{n} \rightarrow G_{n+1}$ induced by $\delta$ is denoted by $\delta_{n}$. A cochain map $f: G \rightarrow G^{\prime}$ is a $\Lambda$-homomorphism such that $f \delta=\delta f$ and $f G_{n} \subseteq G_{n}{ }^{\prime}$; the map $G_{n} \rightarrow G_{n}^{\prime}$ induced by $f$ is denoted by $f_{n}$. The category of cochain complexes and cochain maps is denoted by $\delta g_{\Lambda}$.

If $G$ is a chain complex we set $H(G)=\partial^{-1} 0 / \partial G ; H(G)$ has an induced direct sum decomposition $H(G)=\sum_{n} H_{n}(G)$ where $H_{n}(G)=G_{n} \cap \partial^{-1} 0 / \partial G_{n+1}$. If $f: G \rightarrow G^{\prime}$ is a chain map then $f$ induces homomorphisms $f_{*}: H(G) \rightarrow H\left(G^{\prime}\right)$ and $f_{*}: H_{n}(G) \rightarrow H_{n}\left(G^{\prime}\right)$, where the use of the same symbol $f_{*}$ will cause no confusion. Setting $H(f)=f_{*}$ or $H_{n}(f)=f_{*}$ defines functors $H: \partial \mathcal{G}_{\Lambda} \rightarrow \mathcal{G}_{\Lambda}$ and $H_{n}: \partial \mathcal{G}_{\Lambda} \rightarrow \mathcal{G}_{\Lambda}$.

If $G$ is a cochain complex we set

$$
H(G)=\delta^{-1} 0 / \delta G \text { and } H_{n}(G)=G_{n} \cap \delta^{-1} 0 / \delta G_{n-1}
$$

(this notation differs slightly from the customary but will be more convenient for the abstract portions of this paper); then there is a direct product decomposition $H(G)=\prod_{n} H_{n}(G)$. If $f: G \rightarrow G^{\prime}$ is a cochain map it induces maps $f_{*}: H(G) \rightarrow H\left(G^{\prime}\right)$ and $f_{*}: H_{n}(G) \rightarrow H_{n}\left(G^{\prime}\right)$; setting $H(f)=f_{*}$ and $H_{n}(f)=f_{*}$ defines covariant functors $H: \delta g_{\Lambda} \rightarrow g_{\Lambda}$ and $H_{n}: \delta g_{\Lambda} \rightarrow g_{\Lambda}$.

A chain functor is a functor $K: Q \rightarrow \partial \mathcal{G}_{\Lambda}$, and a cochain functor is a functor $K: Q \rightarrow \delta \mathcal{G}_{\Lambda}$. The functor which to each object $A$ assigns the $n$-dimensional part $(K(A))_{n}$ and to each map assigns the $n$-dimensional part $(K(f))_{n}$ will be denoted by $K_{n}$, where $K$ is a chain or cochain functor; $K_{n}$ is covariant or 
contravariant with $K$. If $K$ is a chain functor there are transformations $\partial_{n}: K_{n} \rightarrow K_{n-1}$ such that $\partial_{n-1} \partial_{n}=0$; if $K$ is a cochain functor there are transformations $\delta_{n}: K_{n} \rightarrow K_{n+1}$ such that $\delta_{n+1} \delta_{n}=0$.

Let $K, L: Q \rightarrow \partial \mathcal{G}_{\Lambda}$ be two chain functors, both covariant or both contravariant; a transformation $f: K \rightarrow L$ is called a chain transformation. A chain transformation $f: K \rightarrow L$ induces transformations $f_{n}: K_{n} \rightarrow L_{n}$ such that $f_{n-1} \partial_{n}$ $=\partial_{n} f_{n}$; and if for each $n$ there is a transformation $f_{n}: K_{n} \rightarrow L_{n}$ such that $f_{n-1} \partial_{n}=\partial_{n} f_{n}$, then there is a chain transformation $f: K \rightarrow L$ inducing the $f_{n}$.

Let $K, L: Q \rightarrow \delta g_{\Lambda}$ be two cochain functors, both covariant or both contravariant; a transformation $f: K \rightarrow L$ is called a cochain transformation. A cochain transform $f: K \rightarrow L$ induces transformations $f_{n}: K_{n} \rightarrow L_{n}$ such that $f_{n+1} \delta_{n}=\delta_{n} f_{n}$; if for each $n$ there is a transformation $f_{n}: K_{n} \rightarrow L_{n}$ such that $f_{n+1} \delta_{n}=\delta_{n} f_{n}$, then there is a cochain transformation $f: K \rightarrow L$ inducing the $f_{n}$.

The central question of this paper is that of the existence and uniqueness of chain or cochain transformations: Let $K, L: a \rightarrow \partial g_{\Lambda}$ (or $K, L: Q \rightarrow \delta G_{\Lambda}$ ) be chain (or cochain) functors, both covariant or contravariant; when is there a chain (or cochain) transformation $f: K \rightarrow L$ ? If $f, g: K \rightarrow L$ are chain (or cochain) transformations, when are the maps $f_{*}, g_{*}: H K \rightarrow H L$ the same? The remainder of this section is devoted to setting up machinery which in connection with the theory of models will enable us to give answers to these questions.

$$
\text { Let }\left\{\begin{array} { l } 
{ K , L : a \rightarrow \partial \mathcal { G } _ { \Lambda } } \\
{ K , L : a \rightarrow \delta \mathcal { G } _ { \Lambda } }
\end{array} \text { be } \left\{\begin{array}{l}
\text { chain } \\
\text { cochain }
\end{array}\right.\right. \text { functors, }
$$

both covariant or both contravariant, and let $f, g: K \rightarrow L$

$$
\begin{aligned}
& \text { be }\left\{\begin{array} { l } 
{ \text { chain } } \\
{ \text { cochain } }
\end{array} \text { transformations. } A \left\{\begin{array}{l}
\text { chain } \\
\text { cochain }
\end{array}\right.\right. \text { homotopy } \\
& D: f \simeq g \text { is a set of transformations }\left\{\begin{array}{l}
D_{n}: K_{n} \rightarrow L_{n+1}, \\
D_{n}: K_{n} \rightarrow L_{n-1}
\end{array}\right.
\end{aligned}
$$

for each $n$, such that

$$
\left\{\begin{array}{l}
\partial_{n+1} D_{n}+D_{n-1} \partial_{n}=f_{n}-g_{n} \\
\delta_{n-1} D_{n}+D_{n+1} \delta_{n}=f_{n}-g_{n}
\end{array}\right.
$$

If a homotopy $D: f \simeq g$ exists, the maps $f_{*}, g_{*}: H K \rightarrow H L$ are equal.

If $G=\sum_{n} G_{n}$ is a chain complex with boundary operator $\partial$, we define the $q$-skeleton ${ }^{q} G$ of $G$ to be the chain complex defined in the following way: ${ }^{q} G$ is the module which is the direct sum of the $G_{n}$ for $n<q,{ }^{q} G=\sum_{n<q} G_{n}$; the boundary operator on ${ }^{q} G$ is the homomorphism which is $\partial_{n}: G_{n} \rightarrow G_{n-1}$ for $n \leq q$, and which is the zero map in dimensions $>q$. If $f: G \rightarrow G^{\prime}$ is a chain map, the $q$-skeleton ${ }^{q} f$ of $f$ is the map ${ }^{q} f:{ }^{q} G \rightarrow{ }^{q} G^{\prime}$ which is equal to $f$ in dimensions $\leq q$ and which is the zero map in dimensions $>q$; the map ${ }^{q} f$ is again a chain 
map. If $K: Q \rightarrow \partial \mathcal{G}_{\Lambda}$ is a chain functor, composing $K$ with the functor " $g$ skeleton" gives a functor which is called the $q$-skeleton of $K$ and is denoted by ${ }^{q} K$.

The $q$-skeleton of a chain complex $G$ is a subcomplex of $G$, and there is thus a natural map, the inclusion map, ${ }^{q} G \rightarrow G$ which is obviously a chain map. There thus arises a natural chain transformation ${ }^{q} K \rightarrow K$ for any chain functor $K$; if $f: K \rightarrow L$ is a chain transformation, its composition with the transformation ${ }^{q} K \rightarrow K$ will be called the $q$-skeleton of $f$ and denoted by ${ }^{q} f:{ }^{q} K \rightarrow L$ (the distinction between the $q$-skeletons of chain maps and of chain transformations must be kept in mind).

If $G=\prod_{n} G_{n}$ is a cochain complex with coboundary operator $\delta$, the $q$ skeleton ${ }^{q} G$ of $G$ is defined to be the direct product of the submodules $G_{n}$ for $n<q,{ }^{q} G=\pi_{n<q} G_{n}$, and the coboundary operator is defined analogously. If $f: G \rightarrow G^{\prime}$ is a cochain map, the $q$-skeleton ${ }^{q} f$ of $f$ is the cochain map ${ }^{q} f:{ }^{q} G \rightarrow{ }^{q} G^{\prime}$ which is equal to $f$ in dimensions $<q$ and is the zero map in dimensions $>q$. If $K: Q \rightarrow \delta g_{\Lambda}$ is a cochain functor, the composition of $K$ with the functor " $q$-skeleton" gives a functor ${ }^{q} K$ which is called the $q$-skeleton of $K$.

The $q$-skeleton of a cochain complex $G$ is a factor complex of $G$, and there is thus a natural map $G \rightarrow^{q} G$ which is clearly a cochain map. There thus arises a natural cochain transformation $L \rightarrow^{q} L$ for any cochain functor; if $f: K \rightarrow L$ is a cochain transformation, the composition of $f$ with the map $L \rightarrow^{q} L$ is called the $q$-skeleton of $f$ and denoted by ${ }^{q} f: K \rightarrow^{q} L$ (again the distinction between the $q$-skeletons of cochain maps and cochain transformations must be kept in mind).

2.2. Models and acyclic functors. Let $(R, \Gamma)$ be a direct or inverse model theory on a category $a$. If $K: Q \rightarrow \partial \mathcal{G}_{\Lambda}$ (or $K: a \rightarrow \delta G_{\Lambda}$ ) is a covariant chain (or cochain) functor, we define $R(K)$ to be the chain functor $a \rightarrow \partial g_{\Lambda}$ (or the cochain functor $Q \rightarrow \delta g_{\Lambda}$ ) whose $n$-dimensional part is $R\left(K_{n}\right)$ (since $K_{n}: Q \rightarrow G_{\Lambda}$, $R\left(K_{n}\right)$ is already defined); if $f: K \rightarrow L$ is a chain (or cochain) transformation of covariant chain (or cochain) functors, we define $R(f)$ to be the chain (or cochain) transformation whose $n$-dimensional part is $R\left(f_{n}\right)$. It is clear that $R\left({ }^{a} K\right)={ }^{q} R(K)$ and $R\left({ }^{a} f\right)={ }^{q} R(f)$.

A functor $K: Q \rightarrow \partial g_{\Lambda}$ is called acyclic in dimension $n$ if there are transformations $\Delta_{n}: K_{n} \rightarrow K_{n+1}, \Delta_{n-1}: K_{n-1} \rightarrow K_{n}$ such that $\partial_{n+1} \Delta_{n}+\Delta_{n-1} \partial_{n}$ is the identity transformation of $K_{n}$ into itself. The functor $K$ is called semi-acyclic in dimension $n$ if to each object $A$ there is a pair of homomorphisms $\Delta_{n}(A)$ : $K_{n}(A) \rightarrow K_{n+1}(A), \quad \Delta_{n-1}(A): K_{n-1}(A) \rightarrow K_{n}(A)$ such that $\partial_{n+1}(A) \Delta_{n}(A)$ $+\Delta_{n-1}(A) \partial_{n}(A)$ is the identity homomorphism of $K_{n}(A)$ into itself.

A functor $K: Q \rightarrow \delta g_{\Lambda}$ is called acyclic in dimension $n$ if there are transformations $\Delta_{n}: K_{n} \rightarrow K_{n-1}, \Delta_{n+1}: K_{n+1} \rightarrow K_{n}$ such that $\delta_{n-1} \Delta_{n}+\Delta_{n+1} \delta_{n}$ is the identity transformation of $K_{n}$ into itself; $K$ is called semi-acyclic in dimension $n$ if for each object $A$ there is a pair of homomorphisms $\Delta_{n}(A), \Delta_{n+1}(A)$ with this property. 
We shall be interested in the condition that for a certain model theory $(R, \Gamma)$ and a certain chain or cochain functor $K$, the functor $R(K)$ be acyclic in some dimension.

Proposition 3. Let $\left(R_{1}, \Gamma_{1}\right)$ and $\left(R_{2}, \Gamma_{2}\right)$ be model theories on a category $a$, both direct or both inverse, and let $(R, \Gamma)$ be their product; let $K$ be a covariant chain or cochain functor on $Q$. If $R_{1}(K)$ and $R_{2}(K)$ are both acyclic (or both semi-acyclic) in dimension $n$, then so is $R(K)$.

The proof is obvious.

Let now $(a, \mathfrak{T}, S)$ be a category with models. A covariant chain functor $K: a \rightarrow \partial g_{\Lambda}$ is said to be acyclic on models in dimension $n$ if for each model $M$ in $\mathfrak{T}$ there is a pair of homomorphisms $\Delta_{n}(M): K_{n}(M) \rightarrow K_{n+1}(M)$, $\Delta_{n-1}(M): K_{n-1}(M) \rightarrow K_{n}(M)$ such that $\Delta_{n-1}(M) \partial_{n}(M)+\partial_{n+1}(M) \Delta_{n}(M)$ is the identity map $K_{n}(M) \rightarrow K_{n}(M)$. A contravariant cochain functor $K: Q \rightarrow \delta \oint_{\Lambda}$ is said to be acyclic on models in dimension $n$ if for each model $M$ in $\mathbb{T}($ there is a pair of maps $\Delta_{n}(M): K_{n}(M) \rightarrow K_{n-1}(M), \Delta_{n+1}(M): K_{n+1}(M) \rightarrow K_{n}(M)$ such that $\Delta_{n+1}(M) \delta_{n}(M)+\delta_{n-1}(M) \Delta_{n}(M)$ is the identity map $K_{n}(M) \rightarrow K_{n}(M)$.

The following proposition is now easy to establish.

Proposition 4. Let $(a, \mathfrak{T}, S)$ be a category with models, and let $(R, \Gamma)$ be the associated direct model theory on $Q,\left(R^{*}, \Gamma^{*}\right)$ be the associated inverse model theory on $Q^{*}$; let $K: Q \rightarrow \partial \mathcal{G}_{\Lambda}$ be a covariant chain functor and $L: Q \rightarrow \delta \mathcal{G}_{\Lambda}$ be a contravariant cochain functor.

If $K$ is acyclic on models in dimension $n$, then $R(K)$ is acyclic in dimension $n$. sion $n$.

If $L$ is acyclic on models in dimension $n$, then $R^{*}(L)$ is acyclic in dimen-

We have defined the concept of functors being acyclic in a particular d1mension; it is more usual that we should have this condition for all dimensions simultaneously.

Let $K: Q \rightarrow \partial g_{\Lambda}$ be a chain functor. If for each dimension $n$ there is a transformation $D_{n}: K_{n} \rightarrow K_{n+1}$ such that $\partial_{n+1} D_{n}+D_{n-1} \partial_{n}=1$ then $K$ is said to be acyclic. Similarly the concept of semi-acyclicity can be defined; and if $(a, \mathfrak{M}, S)$ is a category with models, we can define what is meant by saying $K$ is acyclic or naturally acyclic on models.

If $K: a \rightarrow \delta g_{\Lambda}$ is a cochain functor, we can again define the concept of acyclicity and semi-acyclicity for $K$; if $(a, \mathfrak{T}, S)$ is a category with models, we can define the concepts of acyclicity on models. We have then:

Proposition 4a. Let $(Q, \mathfrak{T}, S)$ be a category with models, and let $(R, \Gamma)$, $\left(R^{*}, \Gamma^{*}\right)$ be the associated direct and inverse model theories. Let $K: Q \rightarrow \partial \mathcal{G}_{\Lambda}$ be a covariant chain functor and $L: Q \rightarrow \delta G_{\Lambda}$ a contravariant cochain functor on $Q$.

If $K$ is acyclic on models, then $R(K)$ is acyclic.

If $L$ is acyclic on models, then $R^{*}(L)$ is acyclia 
2.3. The existence and uniqueness theorems. Throughout this section we will usually make one or another of two hypotheses, which will be called Hypothesis A and Hypothesis B:

Hypothesis A. $a$ is a category, $(R, \Gamma)$ is a direct model theory on $a$, and $K, L: Q \rightarrow \partial g_{\Lambda}$ are covariant chain functors.

Hypothesis B. $a$ is a category, $(R, \Gamma)$ is an inverse model theory on $a$, and $K, L: Q \rightarrow \delta g_{\Lambda}$ are covariant cochain functors.

We begin with theorems giving conditions for the existence and uniqueness of chain or cochain transformations.

TheOREM 5A. Under Hypothesis A, let $f:{ }^{q} K \rightarrow L$ be a chain transformation. If $K_{q+1}$ is representable and $R(L)$ is acyclic in dimension $q$, then $f$ has an extension $f^{\prime}:{ }^{a+1} K \rightarrow L$.

TheOREM 5B. Under Hypothesis B, let $f: K \rightarrow^{q} L$ be a cochain transformation. If $R(K)$ is acyclic in dimension $q$ and $L_{q+1}$ is representable, then $f$ has an extension $f^{\prime}: K \rightarrow^{q+1} L$.

Proof. We give the proof for Theorem 5A only, since the proof of Theorem $5 \mathrm{~B}$ is similar.

It is required to find $f_{q+1}: K_{q+1} \rightarrow L_{q+1}$ so that $\partial_{q+1} f_{q+1}=f_{q} \partial_{q+1}$.

Let $\chi$ be a representation of $K_{q-1}$ and let $\Delta_{i}: R\left(L_{i}\right) \rightarrow R\left(L_{i+1}\right)$ for $i=q, q-1$ be such that $\partial_{q+1} \Delta_{q}+\Delta_{q-1} \partial_{q}=1$. Let then $f_{q+1}=\Gamma\left(L_{q+1}\right) \Delta_{q} R\left(f_{q} \partial_{q+1}\right) \chi$; then a simple computation shows that $f_{q+1}$ has the desired property.

Theorem 6A. Under Hypothesis A, let $f, g: K \rightarrow L$ be chain transformations inducing ${ }^{q} f,{ }^{q} g:{ }^{q} K \rightarrow L$, and let $D:{ }^{q} f \simeq{ }^{q} g$ be a chain homotopy. If $K_{q+1}$ is representable and $R(L)$ is acyclic in dimension $q+1$, then $D$ has an extension $D^{\prime}$ : ${ }^{q+1} f \simeq^{q+1} g$.

THEOREM 6B. Under Hypothesis B, let $f, g: K \rightarrow L$ be cochain transformations inducing ${ }^{q} f,{ }^{q} g: K \rightarrow^{q} L$, and let $D:{ }^{q} \simeq^{q} g$ be a cochain homotopy. If $R(K)$ is acyclic in dimension $q+1$ and $L_{q+1}$ is representable, then $D$ has an extension $D^{\prime}:{ }^{q+1} f \simeq^{q+1} g$.

Proof (for Theorem 6A). We must define a transformation $D_{q+1}: K_{q+1}$ $\rightarrow L_{q+2}$ so that $\partial_{q+2} D_{q+1}=f_{q+1}-g_{q+1}-D_{q} \partial_{q+1}$.

Let $\chi$ be a representation of $K_{q-1}$ and let $\Delta_{i}: R\left(L_{i}\right) \rightarrow R\left(L_{i+1}\right)$ for $i=q, q+1$ be such that $\partial_{q+2} \Delta_{q+1}+\Delta_{q} \partial_{q+1}=1$. Let $D_{q+1}=\Gamma\left(L_{q-1}\right) \Delta_{q+1} R\left(f_{q+1}-g_{q+1}-D_{q} \partial_{q+1}\right) \chi$; then a simple computation shows that the desired relation holds.

As a corollary of Theorems 5 and 6 we obtain:

THEOREM 7A. Under Hypothesis A, let $f:{ }^{q} K \rightarrow L$ be a chain transformation, and let $K_{n}$ be representable for $n>q+1, R(L)$ be acyclic in each dimension $n>q$. Then $f$ has an extension $f^{\prime}: K \rightarrow L$, and if $f^{\prime}, f^{\prime \prime}: K \rightarrow L$ are extensions of $f$ there is 
a chain homotopy $D: f^{\prime} \simeq f^{\prime \prime}$ with $D_{n}=0$ for $n<q$.

Theorem 7B. Under Hypothesis B, let $f: K \rightarrow^{q} L$ be a cochain transformation, and let $R(K)$ be acyclic in every dimension $n>q$, and let $L_{n}$ be representable for $n>q+1$. Then $f$ has an extension $f^{\prime}: K \rightarrow L$, and if $f^{\prime}, f^{\prime \prime}: K \rightarrow L$ are extensions of $f$ there is a cochain homotopy $D: f^{\prime} \simeq f^{\prime \prime}$ with $D_{n}=0$ for $n<q$.

The preceding theorems are essentially contained in Eilenberg and MacLane [2]. We turn now to new theorems which give uniqueness conditions for a chain or cochain map with fewer restrictions on $K$ and $L$; however the existence of maps can no longer be established in general.

Theorem 8A. Under Hypothesis A, let $f, g: K \rightarrow L$ be chain transformations inducing ${ }^{q} f,{ }^{q} g:{ }^{q} K \rightarrow L$, and let $D:{ }^{q} f \simeq{ }^{q} g$ be a chain homotopy. If $K_{n}$ is semirepresentable for $n>q+1$ and $R(L)$ is semi-acyclic in each dimension $n>q$, then $f_{*}=g_{*}: H K \rightarrow H L$.

THEOREM 8B. Under Hypothesis B, let $f, g: K \rightarrow L$ be cochain transformations inducing ${ }^{a} f,{ }^{q} g: K \rightarrow^{q} L$ and let $D:{ }^{q} \simeq^{q} g$ be a cochain homotopy. If $R(K)$ is semi-acyclic in each dimension $n>q$ and $L_{n}$ is semi-representable for $n>q+1$, then $f_{*}=g_{*}: H K \rightarrow H L$.

Proof (for Theorem 8A). Let $A$ be an object of $a$, and let $f^{\prime}=R(f) A$, $g^{\prime}=R(g) A, K^{\prime}=R(K) A, L^{\prime}=R(L) A$. Let $\chi_{n}: K_{n}(A) \rightarrow K_{n}^{\prime}$ for $n \geq q+1$ be such that $\left(\Gamma\left(K_{n}\right) A\right) \chi_{n}: K_{n}(A) \rightarrow K_{n}(A)$ is the identity map; and let $\Delta_{i}^{n}$ : $L_{i}^{\prime} \rightarrow L_{i+1}^{\prime}$ for $i=n, n-1$ be such that $\partial_{n+1} \Delta_{n}^{n}+\Delta_{n-1}^{n} \partial_{n}=1$.

It will suffice to find maps $D_{n}: K_{n}(A) \rightarrow L_{n+1}(A)$ such that $\partial_{n+1}(A) D_{n}$ $+D_{n-1} \partial_{n}(A)=f_{n}(A)-g_{n}(A)$; we will construct inductively maps $D_{n}$ with this property and maps $D_{n}^{\prime}: K_{n}^{\prime} \rightarrow L_{n+1}^{\prime}$ with $\left(\Gamma\left(L_{n+1}\right) A\right) D_{n}^{\prime}=D_{n}\left(\Gamma\left(K_{n}\right) A\right)$. We set $D_{n}=D_{n}(A)$ and $D_{n}^{\prime}=R\left(D_{n}\right) A$ for $n<q$, and assume that $D_{n}, D_{n}^{\prime}$ have been defined with the desired properties for $n<m$. Let

$$
D_{m+1}^{\prime}=\Delta_{m+1}^{m+1}\left(f_{m+1}^{\prime}-g_{m+1}^{\prime}-D_{m}^{\prime}\right) \chi_{m+1}\left(\Gamma\left(K_{m+1}\right) A\right)
$$

and let $D_{m+1}=\left(\Gamma\left(L_{m+2}\right) A\right) D_{m+1}^{\prime} \chi_{m+1}$. Then a simple computation shows that the $D$ and $D^{\prime}$ have the desired properties. Hence the theorem follows by induction.

THeorem 9A. Under Hypothesis A, let $f: K \rightarrow L$ and $g:{ }^{a} L \rightarrow K$ be chain transformations, where $f$ induces ${ }^{a} f:{ }^{q} K \rightarrow L$; let ${ }^{a} i:{ }^{a} K \rightarrow K$ and ${ }^{a} j:{ }^{a} L \rightarrow L$ be the natural maps, and let $D: g^{q} f \simeq^{q} i, E: f g \simeq^{q} j$ be chain homotopies. If both $K_{n}$ and $L_{n}$ are semi-representable for $n>q+1$ and if both $R(K)$ and $R(L)$ are semi-acyclic in each dimension $n>q$, then $f_{*}$ is a natural equivalence of $H K$ and $H L$, i.e., for each object $A, f_{*}(A): H(K(A)) \approx H(L(A))$.

Theorem 9B. Under Hypothesis B, let $f: K \rightarrow L$ and $g: L \rightarrow^{a} K$ be cochain transformations, where $f$ induces ${ }^{q} f: K \rightarrow^{q} L$; let ${ }^{a} i: K \rightarrow^{q} K$ and ${ }^{a} j: L \rightarrow^{q} L$ be the 
natural maps, and let $D: g f \simeq^{q} i, E:{ }^{q} f g \simeq^{q} j$ be cochain homotopies. If both $K_{n}$ and $L_{n}$ are semi-representable for $n>q+1$ and if both $R(K)$ and $R(L)$ are semiacyclic in each dimension $n>q$, then $f_{*}$ is a natural equivalence of $H K$ and $H L$.

Proof (for Theorem 9A). Let $A$ be an object of $Q$, and set $K^{\prime}=K(A)$, $L^{\prime}=L(A), f^{\prime}=f(A)$ and $K^{\prime \prime}=R(K) A, L^{\prime \prime}=R(L) A, f^{\prime \prime}=R(f) A$. It will suffice to show that there are maps $g_{n}^{\prime}: L_{n}^{\prime} \rightarrow K_{n}^{\prime}$ such that $\partial_{n+1} g_{n+1}^{\prime}=g_{n}^{\prime} \partial_{n+1}$, and maps $D_{n}^{\prime}: K_{n}^{\prime} \rightarrow K_{n+1}^{\prime}, E_{n}^{\prime}: L_{n}^{\prime} \rightarrow L_{n+1}^{\prime}$ such that $\partial_{n+1} D_{n}^{\prime}+D_{n-1}^{\prime} \partial_{n}=g_{n}^{\prime} f_{n}^{\prime}-1$ and $\partial_{n+1} E_{n}^{\prime}+E_{n-1}^{\prime} \partial_{n}=f_{n}^{\prime} g_{n}^{\prime}-1$.

We will define inductively maps $g^{\prime}, D^{\prime}, E^{\prime}$ with the above properties and maps $g_{n}^{\prime \prime}: L_{n}^{\prime \prime} \rightarrow K_{n}^{\prime \prime}, \quad D_{n}^{\prime \prime}: K_{n}^{\prime \prime} \rightarrow K_{n+1}^{\prime \prime}$, and $E_{n}^{\prime \prime}: L_{n}^{\prime \prime} \rightarrow L_{n+1}^{\prime \prime}$ such that $\left(\Gamma\left(K_{n}\right) A\right) g_{n}^{\prime \prime}=g_{n}^{\prime}\left(\Gamma\left(L_{n}\right) A\right),\left(\Gamma\left(K_{n+1}\right) A\right) D_{n}^{\prime \prime}=D_{n}^{\prime}\left(\Gamma\left(K_{n}\right) A\right)$, and $\left(\Gamma\left(L_{n+1}\right) A\right) E_{n}^{\prime \prime}$ $=E_{n}^{\prime}\left(\Gamma\left(L_{n}\right) A\right)$.

Let $\chi_{n}: K_{n}^{\prime} \rightarrow K_{n}^{\prime \prime}$ and $\chi_{n}: L_{n}^{\prime} \rightarrow L_{n}^{\prime \prime}$ be such that $\left(\Gamma\left(K_{n}\right) A\right) \chi_{n}=1$ and $\left(\Gamma\left(L_{n}\right) A\right) \psi_{n}=1$; let $\Delta_{i}^{n}: K_{i}^{\prime \prime} \rightarrow K_{i+1}^{\prime \prime}$ and $\mathrm{Z}_{i}^{n}: L_{i}^{\prime \prime} \rightarrow L_{i+1}^{\prime \prime}$ for $i=n, n-1$ and $n>q$ be such that $\partial_{n+1} \Delta_{n}^{n}+\Delta_{n-1}^{n} \partial_{n}=1$ and $\partial_{n+1} Z_{n}^{n}+Z_{n-1}^{n} \partial_{n}=1$.

Define $g_{n}^{\prime}=g_{n}(A), g_{n}^{\prime \prime}=R\left(g_{n}\right) A, D_{n}^{\prime}=D_{n}(A), D_{n}^{\prime \prime}=R\left(D_{n}\right) A, E_{n}^{\prime}=E_{n}(A)$ and $E_{n}^{\prime \prime}=R\left(E_{n}\right) A$ for $n<q$, and assume that these maps are defined for $n<m$ and have the desired properties. We define $g_{m+1}^{\prime \prime}=\Delta_{m g_{m}^{m}}^{m} \partial_{m+1}^{\prime \prime} \psi_{m+1}\left(\Gamma\left(L_{m+1}\right) A\right)$ and $g_{m+1}^{\prime}=\left(\Gamma\left(K_{m+1}\right) A\right) g_{m+1}^{\prime \prime} \psi_{m+1}$, then define

and

$$
\begin{aligned}
& D_{m+1}^{\prime \prime}=\Delta_{m+1}^{m+1}\left(g_{m+1}^{\prime \prime} f_{m+1}^{\prime \prime}-1-D_{m}^{\prime \prime} \partial_{m+1}\right) \chi_{m+1}\left(\Gamma\left(K_{m+1}\right) A\right), \\
& E_{m+1}^{\prime \prime}=\mathrm{Z}_{m+1}^{m+1}\left(f_{m+1}^{\prime \prime} g_{m+1}^{\prime \prime}-1-E_{m}^{\prime \prime} \partial_{m+1}\right) \psi_{m+1}\left(\Gamma\left(L_{m+1}\right) A\right),
\end{aligned}
$$

$$
D_{m+1}^{\prime}=\left(\Gamma\left(K_{m+2}\right) A\right) D_{m+1}^{\prime \prime} \chi_{m+1}, E_{m+1}^{\prime}=\left(\Gamma\left(L_{m+2}\right) A\right) E_{m+1}^{\prime \prime} \psi_{m+1} .
$$

A simple calculation then shows that the new maps have the desired properties, and the theorem is established.

REMARK. It is also possible to establish the existence and uniqueness of chain transformations in yet another way. If in a category with models a system of chain maps or chain homotopies is given on the models, satisfying naturalness conditions similar to those in the definition of acyclicity on models, and if the appropriate representability or semi-representability conditions are satisfied, the existence of the corresponding natural transformations is assured.

2.4. Augmentations. In this section we will define the concept of an augmentation, which will be used later.

Let $G$ be a $\Lambda$-module. For any category $a$, the functor $G$ will be the functor $Q \rightarrow g_{\Lambda}$ which assigns to each space $A$ of $Q$ the module $G$, and which assigns to each map $f$ of $a$ the identity map of $G$. As a functor, $G$ is both covariant and contravariant.

Let $K: Q \rightarrow \partial \mathcal{G}_{\Lambda}$ be a chain functor; $K$ is said to vanish in negative dimensions if $K_{n}=0$ for $n<0$. A similar definition is given for cochain functors.

Let $K: Q \rightarrow \partial \mathcal{G}_{\Lambda}$ be a chain functor which vanishes in negative dimensions. 
An augmentation $(\epsilon, G)$ of $K$ consists of a $\Lambda$-module $G$ and a transformation $\epsilon: K_{0} \rightarrow G$ such that the transformation $\epsilon \partial_{1}: K_{1} \rightarrow G$ is zero, $\epsilon \partial_{1}=0$. If $\widetilde{K}$ is defined by $\widetilde{K}_{n}=K_{n}$ for $n \neq-1, \widetilde{K}_{-1}=G, \tilde{\partial}_{n}=\partial_{n}$ for $n \neq 0,-1$ and $\tilde{\partial}_{0}=\epsilon$, $\tilde{\partial}_{-1}=0$, then $\tilde{K}: a \rightarrow \partial \mathcal{G}_{\Delta}$ is a chain functor, covariant or contravariant with $K$, called the augmented chain functor.

If $K: Q \rightarrow \delta \mathcal{G}_{\Lambda}$ is a cochain functor, then an augmentation $(\epsilon, G)$ of $K$ consists of a $\Lambda$-module $G$ and a transformation $\epsilon: G \rightarrow K_{0}$ such that $\delta_{0} \epsilon=0$. The augmented cochain functor $K$ of $K$ is defined in the obvious way.

\section{Chapter III. Cup products}

In this chapter a special case of products in cohomology is dealt with, as a foundation for the treatment of the exterior product in the de Rham theory. A general theory of products will be developed in a later paper.

3.1. Tensor products. We assume the concept of the tensor product $G_{1} \otimes_{\Lambda} G_{2}$ of two $\Lambda$-modules $G_{1}$ and $G_{2}$, as given in Bourbaki [1]. The subscript $\Lambda$ will be omitted when no confusion can result.

If $G$ and $G^{\prime}$ are cochain complexes in $\delta g_{\Lambda}$, a cochain complex $G \otimes G^{\prime}$ is defined as follows:

$$
\begin{aligned}
\left(G \otimes G^{\prime}\right)_{n} & =\sum_{p+q=n} G_{p} \otimes{ }_{\Lambda} G_{q}^{\prime}, \text { the direct sum; } \\
\delta(u \otimes v) & =\delta u \otimes v+(-1)^{p} u \otimes \delta v, \quad \text { for } u \in G_{p}, v \in G_{q}^{\prime} .
\end{aligned}
$$

If $f: G \rightarrow H, g: G^{\prime} \rightarrow H^{\prime}$ are cochain maps, then $f \otimes g: G \otimes G^{\prime} \rightarrow H \otimes H^{\prime}$ is a cochain map.

If $K, L: Q \rightarrow \delta g_{\Lambda}$ are cochain functors of the same kind, a cochain functor $K \otimes L: Q \rightarrow \delta G_{\Lambda}$ is defined by $(K \otimes L)(A)=K(A) \otimes L(A)$ and $(K \otimes L)(f)$ $=K(f) \otimes L(f)$.

Let $K$ and $L$ vanish in negative dimensions; and let $\epsilon_{K}: G_{K} \rightarrow K_{0}, \epsilon_{L}$ : $G_{L} \rightarrow L_{0}$ be augmentations of $K$ and $L$; then $\epsilon_{K} \otimes \epsilon_{L}: G_{K} \otimes G_{L} \rightarrow K_{0} \otimes L_{0}$ $=(K \otimes L)_{0}$ is an augmentation of $K \otimes L$.

Proposition 10. Let $K, L: Q \rightarrow \delta \mathcal{G}_{\Lambda}$ be cochain functors of the same kind, vanishing in negative dimensions, and let $\epsilon_{K}: G_{1} \rightarrow K_{0}, \epsilon_{L}: G_{2} \rightarrow L_{0}$ be augmentations of $K$ and $L$; let $\epsilon_{K} \otimes \epsilon_{L}$ be the induced augmentation of $K \otimes L$, and let $\tilde{K}, \tilde{L},(K \otimes L)^{-}$be the corresponding augmented functors. Let $(a, \mathfrak{T}, S)$ be a category with models.

If $\widetilde{K}$ and $\tilde{L}$ are acyclic on models, so is $(K \otimes L)^{\sim}$.

Proof. We need only prove the following: Let $C$ and $C^{\prime}$ be cochain complexes vanishing in negative dimensions with augmentations $\epsilon: G \rightarrow G_{0}, \epsilon^{\prime}: G^{\prime} \rightarrow C_{0}^{\prime}$, inducing the augmentation $\epsilon \otimes \epsilon^{\prime}: G \otimes G^{\prime} \rightarrow C_{0} \otimes C_{0}^{\prime}=\left(C \otimes C^{\prime}\right)_{0}$, and let $\tilde{C}, \tilde{C}^{\prime}$ and $\left(C \otimes C^{\prime}\right)^{\sim}$ be the corresponding augmented complexes; if $\widetilde{C}$ and $\widetilde{C}^{\prime}$ are acyclic, so is $\left(C \otimes C^{\prime}\right)^{\text {r }}$. Let $D_{n}: \widetilde{C}_{n} \rightarrow \widetilde{C}_{n-1}, D_{n}^{\prime}: \widetilde{C}_{n}^{\prime} \rightarrow \widetilde{C}_{n-1}^{\prime}$ be the cochain homotopies making $\widetilde{C}, \tilde{C}^{\prime}$ acyclic. We have $\delta_{n-1} D_{n}+D_{n+1} \delta_{n}=1, \delta_{n-1} D_{n}^{\prime}+D_{n+1}^{\prime} \delta_{n}=1$; we 
must define $D_{n}^{\prime \prime}:\left(C \otimes C^{\prime}\right)_{n} \rightarrow\left(C \otimes C^{\prime}\right)_{n-1}^{\sim}$ so that $\delta_{n-1} D_{n}^{\prime \prime}+D_{n+1}^{\prime \prime} \delta_{n}=1$.

First observe that for $g \in G, g^{\prime} \in G^{\prime}$ we have $D_{0} \in g=g$ and $D_{0}^{\prime} \in{ }^{\prime} g^{\prime}=g^{\prime}$.

If $a \in \tilde{C}_{p}, b \in \tilde{C}_{q}^{\prime}, p+q=n$, define

$$
\begin{aligned}
D_{n}^{\prime \prime}(a \otimes b) & =\left(D_{p} a\right) \otimes b & & \text { for } p>0, q \geq 0, \\
& =\epsilon D_{0} a \otimes D_{q}^{\prime} b & & \text { for } p=0, q>0, \\
& =D_{0} a \otimes D_{0}^{\prime} b & & \text { for } p=0, q=0
\end{aligned}
$$

and let $D_{n}^{\prime \prime}=0$ for $n<0$. Then for $p=-1, q=-1$ we have

$$
\delta_{-2} D_{-1}^{\prime \prime}(a \otimes b)+D_{0}^{\prime \prime} \delta_{-1}(a \otimes b)=D_{0} \epsilon a \otimes D_{0}^{\prime \prime} b=a \otimes b ;
$$

for $p=0, q=0$ we have

$$
\begin{aligned}
\delta_{-1} D_{0}^{\prime \prime}(a \otimes b)+D_{1} \delta_{0}(a \otimes b) & =\epsilon D_{0} a \otimes \epsilon^{\prime} D_{0}^{\prime} b+D_{1}\left(\delta_{0} a \otimes b+a \otimes \delta_{0} b\right) \\
& =\epsilon D_{0} a \otimes \epsilon^{\prime} D_{0}^{\prime} b+D_{1} \delta_{0} a \otimes b+\epsilon D_{0} a \otimes D_{1}^{\prime} \delta_{0} b \\
& =\epsilon D_{0} a \otimes\left(\epsilon^{\prime} D_{0}^{\prime}+D_{1}^{\prime} \delta_{0}\right) b+D_{1} \delta_{0} a \otimes b \\
& =\left(\epsilon D_{0}+D_{1} \delta_{0}\right) a \otimes b=a \otimes b ;
\end{aligned}
$$

for $p=0, q>0$ we have

$$
\begin{aligned}
\left(\delta_{q-1} D_{q}^{\prime \prime}+D_{q+1}^{\prime \prime} \delta_{q}\right)(a \otimes b) & \\
& =\delta_{0} \epsilon D_{0} a \otimes D_{q}^{\prime} b+\epsilon D_{0} a \otimes \delta_{q-1} D_{q}^{\prime} b+D_{1} \delta_{0} a \otimes b+\epsilon D_{0} a \otimes D_{q+1}^{\prime} \delta_{q} b \\
& =\epsilon D_{0} a \otimes\left(\delta_{q-1} D_{q}^{\prime}+D_{q+1}^{\prime} \delta_{q}\right) b+D_{1} \delta_{0} a \otimes b \\
& =\left(\epsilon D_{0}+D_{1} \delta_{0}\right) a \otimes b=a \otimes b
\end{aligned}
$$

finally for $p>0, q>0$ we have

$$
\begin{aligned}
\left(\delta_{n-1} D_{n}^{\prime \prime}\right. & \left.+D_{n+1}^{\prime \prime} \delta_{n}\right)(a \otimes b) \\
& =\delta_{p-1} D_{p} a \otimes b+(-1)^{p-1} D_{p} a \otimes \delta_{p} b+D_{p+1} \delta_{p} a \otimes b+(-1)^{p} D_{p} a \otimes \delta_{q} b \\
& =a \otimes b
\end{aligned}
$$

3.2. Cup products. Let $K^{\prime}, K^{\prime \prime}, K: a \rightarrow \delta \mathcal{G}_{\Lambda}$ be cochain functors of the same kind, and $f: K^{\prime} \otimes K^{\prime \prime} \rightarrow K$ a cochain transformation. Then $f$ defines a transformation $f_{*}: H\left(K^{\prime}\right) \otimes H\left(K^{\prime \prime}\right) \rightarrow H(K)$ as follows: If $A$ is in $a$ and $u \in K_{p}^{\prime}(A), v \in K_{q}^{\prime \prime}(A)$ are cocycles $(\delta u=0, \delta v=0)$, then $u \otimes v$ is a cocycle of $K_{p+q}(A)$; and if $a \in K_{p+1}^{\prime}(A), b \in K_{q+1}^{\prime \prime}(A)$ then

$$
(u=\delta a) \otimes(v+\delta b)=u \otimes v+\delta(a \otimes[v+\delta b])+(-1)^{p} \delta(u \otimes b)
$$

so that the cohomology class of $u \otimes v$ depends only on the cohomology classes of $u$ and $v$.

The map $f_{*}: H\left(K^{\prime}\right) \otimes H\left(K^{\prime \prime}\right) \rightarrow H(K)$ will be called a cup product, as will the map $f: K^{\prime} \otimes K^{\prime \prime} \rightarrow K$. 
From Proposition 4A and 10 and Theorems 7B, 8B and 9B we obtain the following:

Theorem 11. Let $(a, \mathfrak{T}, S)$ be a category with models, and let $\left(R^{*}, \Gamma^{*}\right)$ be the associated inverse model theory. Let $K^{\prime}, K^{\prime \prime}, K: Q \rightarrow \delta G_{\Lambda}$ be cochain functors of the same kind ranishing in negative dimensions with augmentations $\epsilon^{\prime}: G^{\prime} \rightarrow K_{0}^{\prime}$, $\epsilon^{\prime \prime}: G^{\prime \prime} \rightarrow K_{0}^{\prime \prime}, \epsilon: G \rightarrow K_{0}$, inducing the augmented functors $\tilde{K}^{\prime}, \tilde{K}^{\prime \prime}, \tilde{K}$. Let $\phi: G^{\prime} \otimes G^{\prime \prime} \rightarrow G$. Assume further that $K^{\prime}$ and $K^{\prime \prime}$ are acyclic on models.

If $K_{n}$ is representable for all $n$, there exists a product $f: K^{\prime} \otimes K^{\prime \prime} \rightarrow K$ such that $f\left(\epsilon^{\prime} \otimes \epsilon^{\prime \prime}\right)=\epsilon \phi$, and $f$ is unique up to cochain homotopy.

If $K_{n}$ is semi-representable for all $n$ and if there are products $f_{i}: K^{\prime} \otimes K^{\prime \prime} \rightarrow K$ such that $f_{i}\left(\epsilon^{\prime} \times \epsilon^{\prime \prime}\right)=\epsilon \phi, i=1,2$, then $f_{1 *}=f_{2 *}: H\left(K^{\prime}\right) \times H\left(K^{\prime \prime}\right) \rightarrow H(K)$.

As a corollary we have:

Corollary. Let $K^{\prime}, K^{\prime \prime}, K, L^{\prime}, L^{\prime \prime}, L: Q \rightarrow \delta G_{\Lambda}$ be cochain functors of the same kind vanishing in negative dimensions and let $\epsilon^{\prime}: G^{\prime} \rightarrow K_{0}^{\prime}, \eta^{\prime}: G^{\prime} \rightarrow L_{0}^{\prime}$, $\epsilon^{\prime \prime}: G^{\prime \prime} \rightarrow K_{0}^{\prime \prime}, \eta^{\prime \prime}: G^{\prime \prime} \rightarrow L_{0}^{\prime \prime}, \epsilon: G \rightarrow K_{0}, \eta: G \rightarrow L_{0}$ be augmentations inducing the augmented functors $\widetilde{K}^{\prime}, \tilde{K}^{\prime \prime}, \widetilde{K}, \widetilde{L}^{\prime}, \tilde{L}^{\prime \prime}, \widetilde{L}$. Let $\phi: G^{\prime} \otimes G^{\prime \prime} \rightarrow G$, and let $(\propto, \mathfrak{T}, S)$ be a category with models. Let $K^{\prime}, K^{\prime \prime}$ be acyclic on models, and $L_{n}$ be semirepresentable for all $n$. Let $f^{\prime}: K^{\prime} \rightarrow L^{\prime}, f^{\prime \prime}: K^{\prime \prime} \rightarrow L^{\prime \prime}, f: K \rightarrow L$ be cochain maps, such that $f_{0}^{\prime} \epsilon^{\prime}=\eta^{\prime}, f_{0}^{\prime \prime} \epsilon^{\prime \prime}=\eta^{\prime \prime}, f_{0} \epsilon=\eta_{0} ;$ and let $g: K^{\prime} \otimes K^{\prime \prime} \rightarrow K, h: L^{\prime} \otimes L^{\prime \prime} \rightarrow L$ be products such that $g\left(\epsilon^{\prime} \otimes \epsilon^{\prime \prime}\right)=\epsilon \phi, h\left(\eta^{\prime} \times \eta^{\prime \prime}\right)=\eta \phi$. Then the diagram

$$
\begin{aligned}
& H\left(K^{\prime}\right) \otimes H\left(K^{\prime \prime}\right) \stackrel{g_{*}}{\rightarrow} H(K)
\end{aligned}
$$

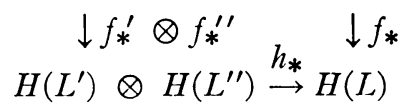

is commutative.

\section{Chapter IV. Singular homology theory}

In this chapter the singular $C^{r}$ homology theory of $C^{r}$ manifolds is described. The independence of the result of the class $r$ is established, as an application of acyclic models.

4.1. The singular functor. Let $a_{r}$ be the category of paracompact manifolds of class $r(r=0,1, \cdots, \infty, \omega)$, and maps of class $r$. For $0 \leq s \leq r$, we define a $C^{s} n$-cube $\sigma$ of a $C^{r}$ manifold $M$ to be a map $\sigma: I^{n} \rightarrow M$ of the unit $n$-cube into $M$, such that $\sigma$ can be extended to a $C^{s}$ map of an open neighborhood of $I^{n}$ (in $n$-space); clearly every $C^{s} n$-cube is a $C^{0} n$-cube (in the future, a $C^{0} n$-cube will be called simply an $n$-cube).

If $\sigma$ is an $n$-cube, $\epsilon=0,1$ and $1 \leq i \leq n$, then we define an $(n-1)$-cube $F_{i}^{\epsilon} \sigma$ by

$$
\left(F_{i}^{\mathrm{e} \sigma}\right)\left(t_{1}, \cdots, t_{n-1}\right)=\sigma\left(t_{1}, \cdots, t_{i-1}, \epsilon, t_{i}, \cdots, t_{n-1}\right) ;
$$

if $1 \leq i \leq n+1$ we define an $(n+1)$-cube $D_{i} \sigma$ by 


$$
\left(D_{i} \sigma\right)\left(t_{1}, \cdots, t_{n+1}\right)=\sigma\left(t_{1}, \cdots t_{i-1}, t_{i+1}, \cdots, t_{n+1}\right) .
$$

,If $\sigma$ is of class $s$, so are $F_{i}^{e} \sigma$ and $D_{i} \sigma$. The operations $F_{i}^{e}$ and $D_{j}$ satisfy the relations

$$
\begin{array}{rlrl}
F_{i}^{e} F_{j}^{n} & =F_{j}^{n} F_{i+1}^{\epsilon} & & i \geq j, \\
D_{i} D_{j} & =D_{j+1} D_{i} & i>j, \\
F_{i}^{\epsilon} D_{j} & =D_{j-1} F_{i}^{\epsilon} & & i>j, \\
F_{i}^{\epsilon} D_{i} & =1, & & \\
F_{i}^{e} D_{j} & =D_{j} F_{i+1}^{\epsilon} & & i<j .
\end{array}
$$

If $G$ is an abelian group, the $C^{s}$ singular chain and cochain complexes with coefficients in $G$ of a $C^{r}$ manifold $M(s \leq r)$ is defined as follows: Let $\tilde{C}_{n}(M)$ be the free abelian group generated by the $C^{s} n$-cubes of $n$, and $D C_{n}(M)$ the subgroup generated by $C^{s} n$-cubes of the form $D_{i} \tau, \tau$ a $C^{s}(n-1)$ cube; let $\tilde{\partial}: \widetilde{C}_{n}(M) \rightarrow \widetilde{C}_{n-1}(M)$ be defined by

$$
\tilde{\partial} \sigma=\sum(-1)^{i+\epsilon} F_{i}^{\epsilon} \sigma .
$$

Then $\tilde{\partial}^{2}=0$ and $\tilde{\partial} D C_{n}(M) \subseteq D C_{n-1}(m)$. Define $C_{n}(M)=\widetilde{C}_{n}(M) / D C_{n}(M)$, and $C(M)=\sum C_{n}(M)$ as the direct sum of the $C_{n}(M)$; let $\partial: C(M) \rightarrow C(M)$ be the map induced by $\tilde{\partial}$. Then $\{C(M), \partial\}$ is a chain complex. We define $C(M ; G)$ as $C(M) \otimes G$ and $C^{*}(M ; G)$ as Hom $(C(M), G)$; then $C(M ; G)$ is a chain complex and $C^{*}(M ; G)$ is a cochain complex called respectively the $C^{*}$ singular chain and cochain complexes of $M$ with coefficients in $G$.

If $f: M \rightarrow N$ is a $C^{r}$ map of $C^{r}$ manifolds, and $\sigma$ is a $C^{s} n$-cube of $M$, then $f \circ \sigma$ is an $n$-cube of $N$; further

$$
\begin{aligned}
& f \circ\left(F_{i}^{e} \sigma\right)=F_{i}^{e}(f \circ \sigma), \\
& f \circ\left(D_{i} \sigma\right)=D_{i}(f \circ \sigma) .
\end{aligned}
$$

Thus $f$ induces chain maps

$$
f_{\boldsymbol{t}}: C(M ; G) \rightarrow C(N ; G), \quad f^{*}: C^{*}(N ; G) \rightarrow C(M ; G) .
$$

The pair $\left\{C(M ; G), f_{t}\right\}$ defines a covariant functor $a_{r} \rightarrow \partial g_{\Lambda}$ and $\left\{C^{*}(M, G)\right.$, $\left.f^{*}\right\}$ defines a contravariant functor $Q_{r} \rightarrow \delta g_{\Lambda}$, which will be called respectively the $C^{s}$ singular chain and cochain functors with coefficients $G$.

Mapping $C_{0}(M)=C_{0}^{T}(M) \rightarrow Z$ (the integers) by $\sum n_{i} \sigma_{i} \rightarrow \sum n_{i}$ defines an augmentation of $C(M)$. If $K$ and $K^{*}$ denote the $C^{*}$ singular chain and cochain functors with coefficients $G$, augmentations

$$
\epsilon: K_{0} \rightarrow G, \quad \epsilon^{*}: G \rightarrow K_{0}^{*}
$$

are defined by the augmentation of $C(M) ; \epsilon$ and $\epsilon^{*}$ will be called the natural augmentations. 
It is known that the $C^{s}$ singular theories all determine the same homology and cohomology theories for all $s$; a proof of this fact will be sketched here.

Let $K$ and $L$ be the $C^{r}$ and $C^{s}$, singular chain functors on $a_{r}, r \geq s$ (a dual proof will apply for the cochain functors); since every $C^{r} n$-cube is a $C^{s}$ $n$-cube, there is a chain transformation $f: K \rightarrow L$.

Let $Q$ be the subcategory of $a_{r}$ formed of all paracompact $C^{r}$ manifolds and inclusion maps $i: M \rightarrow N$ of an oper submanifold $M$ of $N$ into $N$. Then $K, L$ and $f$ are defined on $a$.

Let $\tilde{L}$ be the functor on $a$ defined by letting $L(M)$ be the subcomplex of $C(M ; G)$ generated by $n$-cubes contained in some neighborhood of $M$; in particular, for $r=s, \widetilde{K}$ is also defined. The inclusion maps $k: \widetilde{K} \rightarrow K$ and $l: \tilde{L} \rightarrow L$ are then chain transformations, and $f: K \rightarrow L$ induces a chain transformation $\tilde{f}: \tilde{K} \rightarrow \tilde{L}$, so that

$$
\begin{aligned}
& K \stackrel{f}{\rightarrow} L \\
& k \uparrow \uparrow l \\
& \tilde{K} \underset{\tilde{f}}{\rightarrow} \tilde{L}
\end{aligned}
$$

is a commutative diagram.

By the usual process of subdivision it is easy to see that $k_{*}: H(\tilde{K}) \rightarrow H(K)$ and $l_{*}: H(\widetilde{L}) \rightarrow H(L)$ are isomorphisms. It remains to show that $\tilde{f}_{*}: H(\widetilde{K})$ $\rightarrow H(\tilde{L})$ is an isomorphism; it will then follow that $f_{*}: H(K) \approx H(L)$.

A model theory on $a$ is defined by taking as the set of models $\mathscr{T}$ the set of open cells, and $S(A)$ as the set of coordinate maps $i: M \rightarrow A$ defining the $C^{r}$-structure of the $n$-manifold $A$, where $M \in \mathscr{T}$ is any $n$-cell.

By the definition of $\widetilde{K}$ and $\tilde{L}$, both are representable in the model theory derived from $(\propto, \mathfrak{T}, S)$; by the usual proof of the Homotopy Axiom, $\widetilde{K}$ and $\widetilde{L}$ have acyclic models in all dimensions $\geq 1$; as $K_{0}=L_{0}, f$ has an inverse in dimensions $\leq 0$ and thus by Theorem 7 it follows that $f_{*}$ is an isomorphism.

We state this result as

Proposition 12. If $K$ and $L$ are the $C^{r}$ and $C^{s}$ singular chain or cochain functors on $a_{r}$, the natural inclusion map induces an equivalence

$$
H(K) \approx H(L) .
$$

4.2. Models for the singular theory. In $Q_{r}$, let $\mathfrak{N}_{r}$ be the set of interiors of $n$-cubes, $n=0,1, \cdots$ (note: Int $I^{0}=I^{0}$ ); if $A$ is a $C^{r}$ paracompact manifold, let $S(A)$ be the set of maps $\sigma$ : Int $I^{n} \rightarrow A$ which can be extended to $C^{r}$ maps of some neighborhood of $I^{n}$. The $\sigma \in S(A)$ are in one-one correspondence with the $C^{r}$ singular cubes of $A$.

Proposition 13. Let $G$ be an abelian group, $K$ the $C^{r}$ singular chain (or cochain) functor on $a_{r}$ and $\widetilde{K}$ the augmented functor of $K$. Let $(R, \Gamma)$ be the 
direct (or inverse) model theory associated with $\left(\mathfrak{Q}_{r}, \mathfrak{T}_{r}, S\right)$. Then $\tilde{K}$ is acyclic on models and $K_{n}$ is representable for all $n$.

The proof that $\tilde{K}$ is acyclic on models is the standard proof of the Homotopy Axiom. The proof of the representability is taken from Eilenberg and MacLane [2].

Let $L$ be the functor which to $A \in Q_{r}$ assigns the complex generated by all cubes. Then there is a natural map $\xi: L_{n} \rightarrow K_{n}$ (or $\xi: K_{n} \rightarrow L_{n}$ for the cochain functor) which is onto (or one-one, respectively).

Let $\sigma$ be an $n$-cube. Then define $\eta_{1} \sigma=\left(1-D_{1} F_{1}^{0}\right)\left(1-D_{2} F_{2}^{0}\right) \cdots\left(1-D_{n} F_{n}^{0}\right) \sigma$; then $\eta_{1} \sigma=0$ if $\sigma=D_{i} \tau$ for any $i, \tau$; hence $\eta_{1}$ induces a map $\eta: K_{n} \rightarrow L_{n}$ and $\xi \eta$ is the identity.

Clearly $L_{n}$ is representable; hence by Proposition $1, K_{n}$ is representable.

4.3. Cup products. It follows from Proposition 13 and Theorem 11 that if $K^{(i)}$ is the $C^{r}$ singular cochain functor on $Q_{r}$, with coefficients in $G^{(i)}$ and $\phi: G^{(1)} \otimes G^{(2)} \rightarrow G^{(8)}$ is given, a unique product

$$
\phi_{*}: H\left(K^{(1)}\right) \otimes H\left(K^{(2)}\right) \rightarrow H\left(K^{(3)}\right)
$$

is defined; if $u \in H\left(K^{(1)}(A)\right), v \in H\left(K^{(2)}(A)\right)$, the image $\phi_{*}(u \otimes v)$ will be denoted by $u \cup v$.

Since the product $K^{(1)} \otimes K^{(2)} \rightarrow K^{(3)}$ must commute with maps $f: A \rightarrow B$ it is easy to see that it must be given by a formula; and that it is independent of the class $r$.

The associativity and skew-commutativity of the cup product can be readily established by the methods of acyclic models.

\section{Chapter V. The de Rham theorem}

In this chapter the complex of differential forms on a differentiable manifold is described, and the de Rham theorem is established. For the concept of exterior algebra, see Bourbaki [1].

5.1. The de Rham functor. For the statements of this section, see Gugenheim and Spencer [5].

Let $M$ be a $C^{\infty}$ manifold; by $T(M)$ is denoted the tangent bundle of $M$, which is a $C^{\infty}$ manifold. A cross-section of $T(M)$ is called a vector-field of $M$; the set of $C^{\infty}$ vector fields will be denoted by $V_{M}$, and the set of $C^{\infty}$ functions on $M$ will be denoted by $R(M)$. Then $R(M)$ is a ring containing the real field $R$ (as the constant functions), and $V_{M}$ is an $R(M)$-module. A bracket product $[v, w]$ is defined for $v, w \in V_{M}$; each $v \in V_{M}$ is a differentiation operator on $R(M)$. The dual tangent bundle $T^{*}(M)$ has associated a bundle $T^{*}(M)$ whose fibre is the exterior algebra $\Lambda\left(R^{n}\right)$ (where $M$ is $n$-dimensional); a cross-section of $T^{*}(M)$ is called a differential form of $M$. The set of differential forms of class $\infty$ will be denoted by $\Phi(M) ; \Phi(M)$ has a direct sum decomposition $\Phi(M)=\sum_{0}^{n} \Phi^{p}(M)$, where $\Phi^{p}(M)$ is the set of differential forms of degree $p$ (and $\Phi^{0}(M)=R(M)$ ). 
It is known that there is a natural isomorphism

$$
\Phi^{p}(M) \approx \operatorname{Hom}_{R(M)}\left(\Lambda^{p}\left(V_{M}\right), R(M)\right) .
$$

The set $\Phi(M)$ is an associative ring with product $\wedge$ such that

$$
\phi \wedge \psi=(-1)^{p q} \psi \wedge \phi
$$

for $\phi \in \Phi^{p}(M), \psi \in \Phi q(M)$.

An operation $d: \Phi^{p}(M) \rightarrow \Phi^{p+1}(M)$ is defined by

$$
\begin{aligned}
(d \phi)\left(v_{1} \wedge \cdots \wedge v_{p+1}\right)= & \sum_{i=1}^{p+1}(-1)^{i+1} v_{i}\left(\phi\left(v_{1} \wedge \cdots \hat{\imath} \cdots \wedge v_{p+1}\right)\right. \\
& +\sum_{i<j}(-1)^{i+j+1} \phi\left(\left[v_{i}, v_{j}\right] \wedge v_{1} \cdots \hat{\imath} \cdots \widehat{j} \cdots \wedge v_{p+1}\right) .
\end{aligned}
$$

The operator $d$ is characterized by:

(1) $(d f)(v)=v(f)$ for $f \in \Phi^{0}(M)$,

(2) $d^{2}=0$,

(3) $d(\phi \wedge \psi)=d \phi \wedge \psi+(-1)^{p} \phi \wedge d \psi$ for $\phi \in \Phi^{p}(M)$.

If $f: M \rightarrow N$ is a $C^{\infty}$ map, then $f$ defines a map $f^{\sharp}: \Phi(N) \rightarrow \Phi(M)$, and $f^{\sharp} d=d f^{\sharp}, f^{\sharp}(\phi \wedge \psi)=f^{\sharp} \phi \wedge f^{\sharp} \psi$.

The $\{\Phi(M), d\}$ and $f^{\sharp}$ define a contravariant functor

$$
\Phi: Q_{\infty} \rightarrow \delta G_{R}(R=\text { real field })
$$

which will be called the de Rham functor. The inclusion $R \subseteq \Phi^{0}(M)$ defines an augmentation $\epsilon: R \rightarrow \Phi^{0}$, which will be called the natural augmentation.

If $\sigma$ is a $C^{\infty}$ singular $p$-cube of $M$ and $\phi \in \Phi^{p}(M)$, an integral

$$
\int_{\sigma} \phi=\int_{I^{p}}\left(\sigma^{*} \phi\right)
$$

is defined; this integral has the property that

$$
\int_{D_{i \sigma} \sigma} \phi=0
$$

so that $\int_{\sigma} \phi$, as a function of $\sigma$, defines a linear map $C_{p}(M) \rightarrow R$, that is, $\int \phi$ is a cochain of $M$, so that $\int$ is a map

$$
\int: \Phi^{p}(M) \rightarrow C^{p}(M ; R)
$$

The classical theorem of Stokes states that

$$
\int_{\partial c} \phi=\int_{c} d \phi
$$


so that $\int$ is a cochain map, which then defines a cochain transformation (called the Stokes map)

$$
\int: \Phi \rightarrow C^{*}
$$

where $C^{*}$ is the $C^{\infty}$ singular cochain functor with coefficients in the real field $R$; if $\epsilon^{*}: R \rightarrow C_{0}^{*}, \epsilon: R \rightarrow \Phi^{0}$ are the natural augmentations it is easy to see that $\int \epsilon=\epsilon^{*}$.

The exterior product $\wedge$ defines a product $\pi_{1}: \Phi \otimes \Phi \rightarrow \Phi$, and the product in the real field defines a map $\pi_{2}: R \times R \rightarrow R$; then we have $\pi_{1}(\epsilon \otimes \epsilon)=\epsilon \pi_{2}$. Thus $\wedge$ is a cup product associated with $R \otimes R \rightarrow R$. The product $H(\Phi) \otimes H(\Phi)$ $\rightarrow H(\Phi)$ induced by $\wedge$ will also be denoted by $\wedge$.

5.2. Models for the de Rham functor. We define $\mathfrak{T}_{R}$ to be the set of all open cells, considered as $C^{\infty}$ manifolds; and for a $C^{\infty} n$-manifold $A, S_{R}(A)$ is to be the set of all inclusion maps $i: M \rightarrow A$ of $n$-cells $M$ as open submanifolds of $A$. Then $\left(\mathfrak{Q}_{\infty}, \mathfrak{M}_{R}, S_{R}\right)$ is a category with models.

Proposition 14. Let $\tilde{\Phi}$ be the augmented functor of $\Phi$. Then $\tilde{\Phi}$ is acyclic on models for $\left(\mathfrak{Q}_{\infty}, \mathfrak{M}_{R}, S_{R}\right)$.

For a particularly elegant proof of this fact (sometimes called the Poincaré Lemma), see Gugenheim and Spencer [5, \$4].

Proposition 15. Each $\Phi^{p}$ is semi-representable for the inverse model theory associated with $\left(a_{\infty}, \mathfrak{T}_{R}, S_{R}\right)$.

Let $R$ be the associated model theory; then $R\left(\Phi^{p}\right)(A)=\sum_{\sigma \in S_{(A)}} \Phi^{p}\left(M_{\sigma}\right)$ where $M_{\sigma}$ runs over the open coordinate neighborhoods of $A$. Let $\left\{U_{\alpha}\right\}$ be a locally finite covering of $A$ by coordinate cells (which exists since each $A \in a_{\infty}$ is paracompact), and let $\left\{\phi_{\alpha}\right\}$ be a partition of unity carried by $\left\{U_{\alpha}\right\}$, each $\phi_{\alpha}$ being a $C^{\infty}$ function on $A$ vanishing outside $U_{\alpha}$, with $\sum \phi_{\alpha}=1$. Then if $\omega=\left\{\omega_{\sigma}\right\}$ is a vector of $R\left(\Phi^{p}\right)(A)$, let $\chi(A) \omega=\sum \phi_{\alpha} \omega_{\alpha}$. It follows that $\chi(A) \Gamma(A)$; $=1$, so that $\Phi^{p}$ is semi-representable.

REMARK. $\Phi^{p}$ is not representable, so that the concept of semi-representability is essential in the treatment of $\Phi$.

\section{3. de Rham's theorem.}

THEOREM 16. Let $C^{*}$ be the $C^{\infty}$ singular cochain functor with real coefficients on $a_{\infty}$ and $\Phi$ be the de Rham functor, where $a_{\infty}$ is the category of paracompact $C^{\infty}$ manifolds. The Stokes map $\int: \Phi \rightarrow C^{*}$ induces an isomorphism $\int_{*}: H(\Phi) \approx H\left(C^{*}\right)$; if $\alpha, \beta \in H(\Phi(A))$, then

$$
\int_{*}(\alpha \wedge \beta)=\left(\int_{*} \alpha\right) \cup\left(\int_{*} \beta\right) .
$$

It follows from Proposition 2 and Proposition 3 that for some model 
theory $(R, \Gamma)$ both $\Phi^{p}$ and $C^{p}$ are semi-representable for all $p$, and that $R(\tilde{\Phi}), R\left(\tilde{C}^{*}\right)$ are acyclic where $\widetilde{\Phi}, \tilde{C}^{*}$ are the augmented functors of $\Phi, C^{*}(R$ is the product of the model theories described in $\$ 5.2$ and $\$ 4.2)$. The map $\int: \tilde{\Phi} \rightarrow \widetilde{C}^{*}$ is an isomorphism in dimension -1 (i.e., $\int: R \approx R$ ), and thus $\int_{*}$ is an isomorphism by Theorem $9 \mathrm{~B}$. By Theorem 11, $\int_{*}$ preserves products. Hence de Rham's theorem follows.

\section{BIBLIOGRAPHY}

1. N. Bourbaki, Algèbre, Chapitre III, Algèbre multilinéaire, Paris.

2. S. Eilenberg and S. MacLane, Acyclic models, Amer. J. Math. vol. 75 (1953) pp. 189-199.

3. S. Eilenberg and N. Steenrod, Foundations of algebraic topology, Princeton.

4. V. K. A. M. Gugenheim and J. Moore, Acyclic models and fibre spaces, Trans. Amer. Math. Soc. vol. 85 (1957) pp. 265-306.

5. V. K. A. M. Gugenheim and D. C. Spencer, Chain homotopy and the de Rham theory, Proc. Amer. Math. Soc. vol. 7 (1956) pp. 144-152.

UNIVERSITY OF WASHINGTON, Seatrle Wash. 